\title{
Observation of Peculiar Rashba-Type Spin-Split Band on Bi(111) Surface by High-Resolution Spin- and Angle-Resolved Photoemission Spectroscopy*
}

\author{
Hirokazu Miyahara, Takamasa Maegawa, Kenta Kuroda, and Akio Kimura \\ Graduate School of Science, Hiroshima University, Higashi-Hiroshima 739-8526, Japan \\ Koji Miyamoto and Hirofumi Namatame \\ Hiroshima Synchrotron Radiation Center, Hiroshima University, Higashi-Hiroshima 739-0046, Japan \\ Masaki Taniguchi \\ Graduate School of Science, Hiroshima University, Higashi-Hiroshima 739-8526, Japan and \\ Hiroshima Synchrotron Radiation Center, Hiroshima University, Higashi-Hiroshima 739-0046, Japan \\ Taichi Okuda ${ }^{\dagger}$ \\ Hiroshima Synchrotron Radiation Center, Hiroshima University, Higashi-Hiroshima 739-0046, Japan. \\ (Received 31 January 2012; Accepted 10 April 2012; Published 27 April 2012)
}

\begin{abstract}
Spin polarized surface states of $\mathrm{Bi}(111)$ film fabricated on $\mathrm{Si}(111)$ surface have been investigated by means of high-resolution spin-resolved photoemission spectroscopy. In contrast to the Rashba-type spin-split ideal twodimensional electron gas in which the spin orientation is locked in-plane and tangential to the electron momentum, significant out-of-plane spin polarization has been observed in the surface states which are forming the hole pocket. The out-of-plane spin polarization is anti-symmetric with respect to $\bar{\Gamma}-\bar{M}$ line and vanishes at the line suggesting that the out-of-plane polarization is derived from the three-fold symmetry of the Bi crystal. The magnitude of the out-of-plane spin components depends on the condition of Bi film fabrication, which is consistent with the recent x-ray diffraction results suggesting the existence of double domains rotating $180^{\circ}$ each other on the $\operatorname{Bi}(111)$ film grown on $\mathrm{Si}(111)$ surface. [DOI: 10.1380/ejssnt.2012.153]
\end{abstract}

Keywords: Spin and angle resolved photoemission; Bismuth; Metallic films

\section{INTRODUCTION}

Due to the breaking of space inversion symmetry, at surfaces or interfaces spin degeneracy of electronic states can be lifted off by spin-orbit interaction(SOI) and spinpolaized surface states emerge even in the non-magnetic materials. This spin-polarized surface states by the socalled Rashba effect [1] have been directly observed in several simple metals or alloys containing heavy atoms having large SOI, by high-resolution angle-resolved photoemission spectroscopy (ARPES) and also by spin-resolved ARPES, recently. For example, spin-split surface states have been observed on $\mathrm{Au}(111)$ [2, 3], $\mathrm{Sb}(111)$ [4], Bibased surface alloys such as $\mathrm{BiAg}_{2}[5,6], \mathrm{BiCu}_{2}$ [7] and some reconstructed semiconductor surfaces with heavy elements such as $\mathrm{Au} / \mathrm{Si}(553)$ [8], $\mathrm{Au} / \mathrm{Si}(557)$ [9], $\mathrm{Tl} / \mathrm{Si}(111)$ [10], and $\mathrm{Pb} / \mathrm{Ge}(111)$ [11].

Bismuth is the heaviest nonradioactive metal and has been investigated extensively because of its interesting physical properties. The bulk crystal has a rhombohedral structure with space group $\mathrm{R} \overline{3} \mathrm{~m}$. It has been reported that the high-quality $\mathrm{Bi}(111)$ single crystal film can be fabricated on the clean $\mathrm{Si}(111)$ surface [12] in which the Bi atoms are stacked in bilayer and form honeycomb structure. At the $\mathrm{Bi}(111)$ surface, characteristic Fermi sur-

\footnotetext{
*This paper was presented at the 6th International Symposium on Surface Science - Towards Nano, Bio and Green Innovation-, Tower Hall Funabori, Tokyo, Japan, December 11-15, 2011, and won the Best Poster Award.

$\dagger$ Electronic address: okudat@hiroshima-u.ac.jp
}

face (FS) features, hexagonal electron pocket around $\bar{\Gamma}$ point and six fold petal-like hole pockets lying along $\bar{\Gamma}-\bar{M}$ line, have been observed by ARPES measurement [13]. It has been demonstrated by a first principles calculation that these FS features are ascribed to Rashba-type spin-splitting driven by the strong SOI of Bi atom [14]. The in-plane spin-polarization of these surface states at around Fermi energy $\left(E_{F}\right)$ was also measured directly by SARPES and the spin splitting was confirmed to originate from the Rashba-type spin-split $[15,16]$. Very recently, the existence of giant out-of-plane spin components has been reported in the surface hole-pocket, which suggests the deviation from the Rashba effect of ideal two-dimensional electron gas in $\mathrm{Bi}(111)$ [17]. However, the observed SARPES spectra were quite broad and even the peak positions of the surface states were not well resolved probably due to the poor momentum resolution $\left(\Delta k>0.05 \AA^{-1}\right)$ in the measurement. In addition, the origin of the out-of-plane spin component has not been well understood.

In this report we present the results of high-resolution SARPES observation of spin-structure at the $\mathrm{Bi}(111)$ surface using high-efficient SARPES machine [18] constructed at beamline BL-9B in Hiroshima Synchrotron Radiation Center (HSRC). With the higher resolutions $\left(\Delta E \sim 20 \mathrm{meV}\right.$ and $\left.\Delta k \sim 0.02 \AA^{-1}\right)$, both in-plane and out-of-plane spin components have been clearly observed on the surface states near the FS forming the holepocket. Significant out-of-plane spin polarization having anti-symmetric spin polarization against $\bar{\Gamma}-\bar{M}$ line is confirmed in the surface state while the in-plane spin component shows typical Rashba-type helical spin structure. It is found that the magnitude of the out-of-plane spin po- 


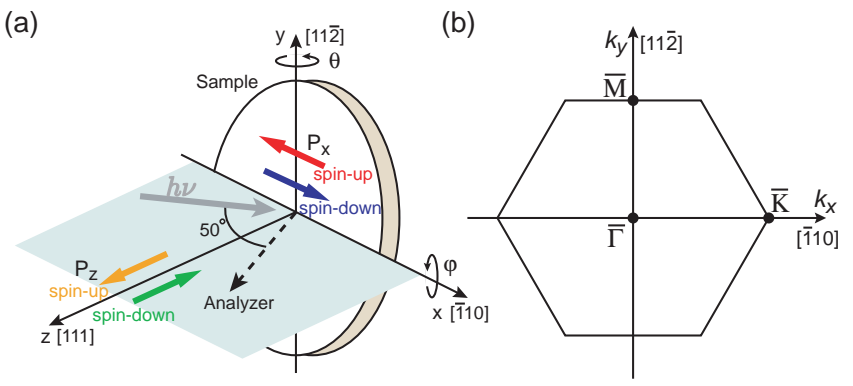

FIG. 1: (a) Schematic illustration of experimental setup and measured spin components, in-plane $\left(P_{x}\right)$ and out-of-plane $\left(P_{z}\right)$. (b) Schematic illustration of surface Brillouin zone (SBZ).

larization depends on the condition of the film fabrication implying that the out-of-plane spin component is derived from the three-fold crystal symmetry of the system.

\section{EXPERIMENTAL}

$\operatorname{Bi}(111)$ thin film was grown on the $\operatorname{Si}(111) 7 \times 7$ surface cleaned by resistive heating at $1500 \mathrm{~K}$ by depositing $\mathrm{Bi}$ at room temperature using a Knudsen cell. After the deposition, the film was annealed at $400 \mathrm{~K}$ to obtain flat surface with large terraces[12]. The quality of the film was checked by a sharp $1 \times 1$ low energy electron diffraction pattern and the clear surface states in spin-integrated ARPES measurement. No detectable contamination was observed by Auger electron spectroscopy. ARPES and SARPES measurements were performed at newly constructed end-station named Efficient SPin REsolved SpectroScOpy(ESPRESSO) [18] at the beamline BL-9B in HSRC. In the beamline one can use high quality variable polarized synchrotron radiation light from the APPLE-II type undulator with a quasi-periodic magnetic array as well as the light from high-flux rare gas (He and $\mathrm{Xe)}$ discharge lamps. In the present study, data were taken with the light from the He discharge lamp (He I $\alpha$, $\mathrm{h} \nu=21.22 \mathrm{eV}$ ) at the sample temperature of $80 \mathrm{~K}$. The ESPRESSO end-station consists of a hemispherical photoelectron analyzer (VG-SCIENTA R4000) combined with home-made high-efficient spin-polarimeter based on the very-low energy electron diffraction (VLEED) using passivated $\mathrm{Fe}(100)$ film by pure oxygen [19]. In the system, both high-quality spin-integrated ARPES mapping by photoelectron multi detection system and spin-polarized ARPES measurement at specific angles can be done with high-resolution. The details of the ESPRESSO machine are described elsewhere [18]. The energy and angular resolutions of ARPES and SARPES measurements of present study were set at $10 \mathrm{meV}, 0.1^{\circ}$ and $20 \mathrm{meV}, \pm 0.35^{\circ}$, respectively. The effective Sherman function, the spin resolving power, of the target of spin polarimeter in the present study was estimated as 0.30 .

Figure 1(a) shows the experimental geometry of the measurements. The angle between light incidence and the electron analyzer was fixed to $50^{\circ}$. In the present setup one can measure spin components along $x$ and $z$ directions of analyzer coordinate which correspond to in-plane $\left(P_{x}\right)$
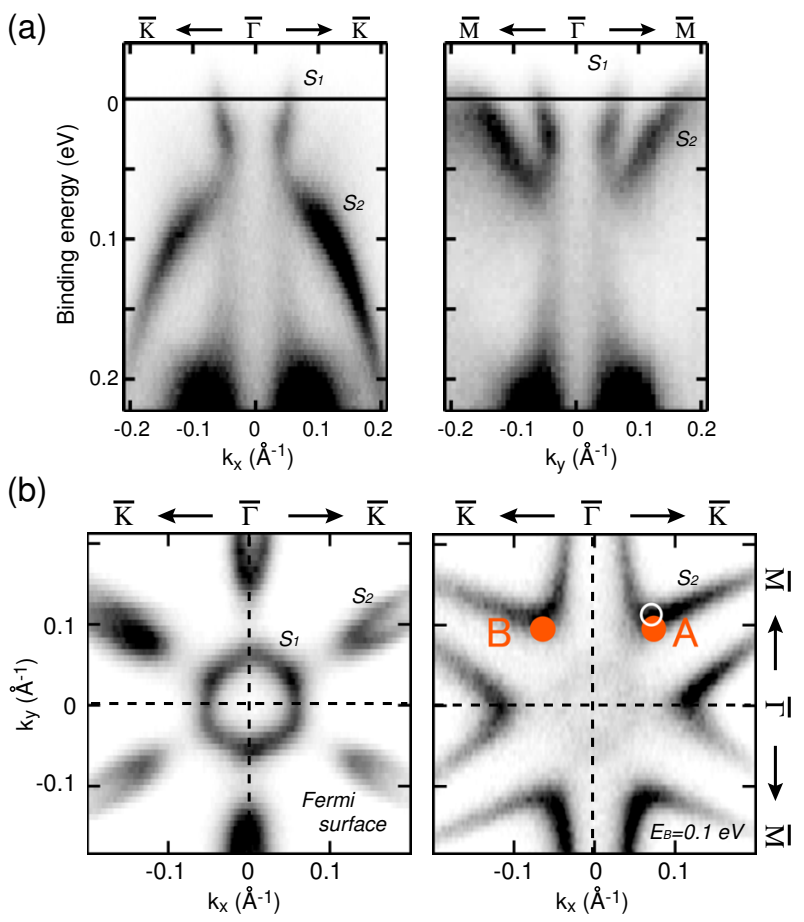

FIG. 2: (a)Spin-integrated ARPES results of Bi(111) film along $\bar{\Gamma}-\overline{\mathrm{K}}$ and $\bar{\Gamma}-\overline{\mathrm{M}}$ directions. Surface states $S_{1}$ and $S_{2}$ are clearly seen. (b) Constant energy contour of surface sates of $\mathrm{Bi}(111)$ film at $E_{F}$ and $E_{B}=0.10 \mathrm{eV}$.

and out-of-plane $\left(P_{z}\right)$ spin components of sample coordinate in the normal emission configuration. Note that the sample coordinate can be different from analyzer coordinate when we rotate the $\operatorname{polar}(\theta)$ and tilt $(\phi)$ angles of the sample. Because of the small rotation angle both in polar and tilt $\left(|\theta|,|\phi|<4^{\circ}\right)$ in the present study, however, the observed spin component along $x$ and $z$ direction of the spin-polarimeters almost corresponds to $P_{x}$ and $P_{z}$ of sample coordinate. Fig. 1(b) shows the surface Brillouin zone (SBZ) at $\mathrm{Bi}(111)$ surface.

\section{RESULTS \& DISCUSSION}

Figure 2(a) shows the results of spin-integrated ARPES measurement in $\bar{\Gamma}-\overline{\mathrm{K}}$ and $\bar{\Gamma}-\overline{\mathrm{M}}$ lines. Surface states $S_{1}$ and $S_{2}$ are clearly seen as reported in previous studies [13]. These $S_{1}$ and $S_{2}$ states form characteristic hexagonal electron pocket surrounding $\bar{\Gamma}$ point and six petallike hole pockets extended along the $\bar{\Gamma}-\bar{M}$ line as shown in the left panel of Fig. 2(b). No quantum-well state has been observed since our Bi film was quite thick and almost bulk like. As the binding energy $\left(E_{B}\right)$ increases, the size of petal-like hole pocket grows up and the adjacent petals merge to each other and eventually deform into the wedge structure at $E_{B} \sim 0.10 \mathrm{eV}$ as seen in the right panel of Fig. 2(b). Note that the apexes of the petal-like structure at Fermi level are at $\bar{\Gamma}-\bar{M}$ line while those of the wedge structure at $E_{B} \sim 0.10 \mathrm{eV}$ are at $\bar{\Gamma}-\overline{\mathrm{K}}$ line.

To unveil the spin characters of the $S_{2}$ state we have performed the SARPES measurement at around the corner of this wedge structure. The observed k-points, 
$\mathrm{A}\left(k_{x}, k_{y}\right)=\left(0.073 \AA^{-1}, 0.095 \AA^{-1}\right)$ and $\mathrm{B}\left(k_{x}, k_{y}\right)=$ $\left(0.064 \AA^{-1}, 0.095 \AA^{-1}\right)$, are marked with filled circles in the right panel of Fig. 2(b). We have observed in-plane spin component along $x(\bar{\Gamma}-\overline{\mathrm{K}})$ direction and out-ofplane component along $z$ direction $(\langle 111\rangle)$ as depicted in Figs. 3(a) and 3(b). The SARPES spectra in the spin-up and -down channels are denoted with triangles pointing up and down, respectively in the figures. As demonstrated in Fig. 3(b) both at point A and point B the spin-up spectra show the larger intensity than those in the spin-down channel for the in-plane spin component being consistent with the anti-clockwise tangential spin polarization to the electron momenta (helical spin polarization) which is derived from the Rashba effect of ideal two-dimensional electron gas. The absolute values of in-plane spin polarization at $\mathrm{A}$ and $\mathrm{B}$ are, however, different from each other, where spin-polarization of point $\mathrm{A}$ is much larger than that of point $\mathrm{B}$. This deviation from the ideal helical spin structure has also been reported by Takayama et al. from the SARPES observation of $S_{2}$ surface state at the $E_{F}$ and interpreted as oscillating spin-polarization implying the breaking of time-reversal symmetry in the $\mathrm{Bi}(111)$ film[17]. However, generally, the photoemission final state effect can produce the variation of spin-polarization at different $\mathbf{k}$ point and further theoretical and experimental investigations are required to solve this issue.

Deviating from the expected spin-polarization of ordinary Rashba effect, very large out-of-plane spin component is observed both at points A and B as in Fig. 3(a). Interestingly the sign of spin polarization at $\mathrm{A}$ is opposite to that of at B. As indicated in Fig. 2(b), the two wedge structures evolve into a petal-like structure at $E_{F}$ and have density of state at along $\bar{\Gamma}-\bar{M}$ line. Since the sign of out-of-plane spin polarization is opposite between adjacent wedge structures, it is expected that the spinpolarization vanishes in the $\bar{\Gamma}-\overline{\mathrm{M}}$ line and the disappearance of the spin-polarization has been actually observed at the $\bar{\Gamma}-\bar{M}$ line (not shown here). Thus, the difference in absolute values of out-of-plane spin polarization between $\mathrm{A}$ and $\mathrm{B}$ points is probably due to the final state effect. The observed large out-of-plane spin component and its sign-reversal against $\bar{\Gamma}-\bar{M}$ line is consistent with the previous report in which the sign-reversal of out-ofplane spin component of petal-like FS against the $\bar{\Gamma}-\bar{M}$ line has been observed [17]. As discussed in the previous paper, the peculiar out-of-plane spin component in the $\mathrm{Bi}(111)$ film is probably derived from the three-fold symmetry of the Bi crystal structure.

Here we remark that the out-of-plane spin component is not always observed but dependent on film quality. Figure 4 shows the SARPES spectra of Bi film fabricated in different growth condition (evaporation rate, annealing temperature) taken at the point indicated with open circle in Fig. 2(b). In this case, out-of-spin polarization is almost zero while the in-plane spin polarization is still positively polarized [20].

According to the recent surface x-ray diffraction measurement, Bi film grown on $\mathrm{Si}(111)$ surface can contain two different domains in which the stacking of the $\mathrm{Bi}$ film having three fold symmetry is $180^{\circ}$ rotated to each other [21]. The variation of the out-of-spin component in different Bi film can probably be attributed to the different ratio of the two domains since the photoemission mea-

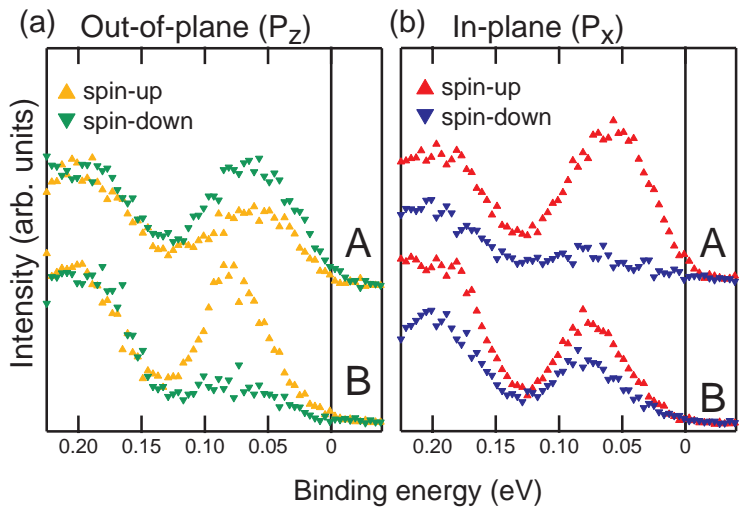

FIG. 3: Spin-resolved energy distribution curve of (a) out-ofplane $\left(P_{z}\right)$ and $(\mathrm{b})$ in-plane $\left(P_{x}\right)$ spin components at $\mathrm{A}$ and $\mathrm{B}$ points in Fig.2 (b).

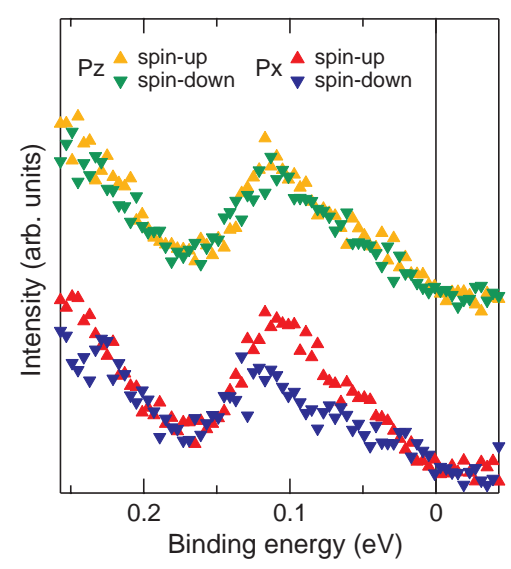

FIG. 4: SARPES spectra of out-of-plane and in-plane spin components observed near the A point (white open circle) in Fig. 2(b) for differently prepared sample compared to that measured in Fig. 3.

surement gives the averaged information from the sample surface illuminated by the excitation light with finite spot $\operatorname{size}(\sim \phi 5 \mathrm{~mm}$ in the present measurement). In other words, the observed variation of out-of-plane spin polarization is in agreement with the hypothesis that the outof-plane spin component is originated from the three-fold symmetry of the crystal structure of $\operatorname{Bi}(111)$ film since the signs of out-of-plane spin polarization are opposite between the two different domains and cancel out. Robustness of in-plane spin component regardless of the film condition also supports the idea since the in-plane spin component is not affected by the three-fold symmetry of the Bi film structure.

\section{CONCLUSION}

We have observed the Rashba-type spin-split band on the surface of $\mathrm{Bi}(111)$ thin film grown on $\mathrm{Si}(111)$ by highresolution SARPES machine with high efficient VLEEDtype spin polarimeter. In addition to the in-plane spin polarization, significant out-of-plane spin polarization whose sign is reversed across the $\bar{\Gamma}-\overline{\mathrm{M}}$ line has been observed for 
the $S_{2}$ states being consistent with the previous SARPES results. This peculiar spin structure deviating from the ordinary Rashba-type spin-split, would be derived by the three-fold symmetry of the crystal structure of $\mathrm{Bi}(111)$ similar to the observed out-of-plane spin polarization in some topological insulators [22]. Furthermore, we have found that the magnitude of out-of-plane spin polariza- tion depends on the condition of Bi film fabrication, which is consistent with the hypothesis that the out-ofplane spin is derived from the three-fold symmetry of the $\mathrm{Bi}(111)$ crystal and the recent x-ray diffraction observation which suggests the existence of double $\mathrm{Bi}(111)$ domains rotating $180^{\circ}$ each other on the $\mathrm{Bi}$ film grown on $\operatorname{Si}(111)$ surface [21].
[1] Y. A. Bychkov and E. I. Rashba, JETP Lett. 39, 78 (1984).

[2] S. LaShell, B. A. McDougall, and E. Jensen, Phys. Rev. Lett. 77, 3419 (1996).

[3] M. Hoesch et al., Phys. Rev. B 69, 241401 (2004).

[4] K. Sugawara et al., Phys. Rev. Lett. 96, 046411 (2006).

[5] C. R. Ast et al., Phys. Rev. Lett. 98, 186807 (2007).

[6] F. Meier et al., Phys. Rev. B 77165431 (2008).

[7] L. Moreschini et al., Phys. Rev. B 80, 035438 (2009).

[8] J. N. Crain et al., Phys. Rev. B 69, 125401 (2004).

[9] T. Okuda et al., Phys. Rev.B 82, 161410 (2010).

[10] K. Sakamoto et al., Phys. Rev. Lett. 102, 096805 (2009).

[11] K. Yaji et al., Nat. Commun. 1, 17(2010).

[12] T. Nagao et al., Phys. Rev. Lett. 93, 105501 (2004).

[13] C. R. Ast et al., Phys. Rev. Lett. 87, 177602 (2001).

[14] Y. M. Koroteev et al., Phys. Rev. Lett. 93, 046403 (2004).

[15] T. Hirahara et al., Phys. Rev. B 76, 153305 (2007).
[16] T. Hirahara et al., New J. Phys. 10, 083038 (2008).

[17] A. Takayama et al., Phys. Rev. Lett. 106, 166401 (2011).

[18] T. Okuda et al., Rev. Sci. Instrum. 82, 103302 (2011).

[19] T. Okuda et al. Rev. Sci. Instrum. 79, 123117 (2008) and references there in.

[20] Comparing the data of in-plane component at point $\mathrm{A}$ in Fig. 3(b) and Fig. 4, the absolute spin polarization and the spectral shapes are different to each other. Although the reason of the differneces is not clear the slightly different measurement position (filled and open circles in the Fig. 2(b) ) and different measurement geometry $\left(\phi=2.6^{\circ}\right.$, $\theta=2.2^{\circ}$ in Fig. 3(b) and $\phi=3.2^{\circ}, \theta=2^{\circ}$ in Fig. 4) can affect the observed SARPES spectra because of the slightly different initial and final states between them.

[21] T. Shirasawa et al., Phys. Rev. B 84, 075411 (2011).

[22] S. Souma et al., Phys. Rev. Lett. 106, 216803 (2011). 